\title{
Association between Helicobacter pylori Infection and Diabetes: A Cross-Sectional Study in China
}

\author{
Sailimai Man $\mathbb{D},{ }^{1,2,3}$ Yuan Ma, ${ }^{2,3}$ Cheng Jin, ${ }^{2,3}$ Jun Lv, ${ }^{1,3}$ Mingkun Tong, ${ }^{2,3}$ Bo Wang $\mathbb{D},{ }^{2,3}$ \\ Liming Li $D^{1,3}$ and Yi Ning ${ }^{2,3}$ \\ ${ }^{1}$ Department of Epidemiology and Biostatistics, School of Public Health, Peking University Health Science Center, \\ Beijing 100191, China \\ ${ }^{2}$ Meinian Institute of Health, Beijing 100191, China \\ ${ }^{3}$ Peking University Health Science Center Meinian Public Health Institute, Beijing 100191, China
}

Correspondence should be addressed to Bo Wang; paul@meinianresearch.com, Liming Li; lmlee@bjmu.edu.cn, and Yi Ning; yi.ning@meinianresearch.com

Sailimai Man and Yuan Ma contributed equally to this work.

Received 15 May 2020; Revised 14 August 2020; Accepted 25 August 2020; Published 25 September 2020

Academic Editor: Patrizio Tatti

Copyright (c) 2020 Sailimai Man et al. This is an open access article distributed under the Creative Commons Attribution License, which permits unrestricted use, distribution, and reproduction in any medium, provided the original work is properly cited.

Background. Studies suggest an association between $H$. pylori infection and extragastrointestinal disease. Limited studies provided conflicting results on the association between $H$. pylori infection and diabetes. The present study was aimed at examining the association between $H$. pylori infection and diabetes in a large health checkup population in China. Methods. A cross-sectional study was conducted; participants who attended health checkups at Beijing MJ Health Screening Center during 2017-2018 were included. $H$. pylori infection was diagnosed by ${ }^{13} \mathrm{C}$-urea breath test. Multivariate logistic regression analysis was performed to evaluate the association between $H$. pylori infection and diabetes. Results. The mean age of 13,397 participants was $43.8 \pm 12$ years. The prevalence of $H$. pylori infection and diabetes was $28.2 \%$ and $8.1 \%$, respectively. The prevalence of diabetes was higher among $H$. pylori-positive participants compared with their counterparts $(8.9 \%$ vs $7.8 \%, p=0.05)$. After adjustment of age, sex, family history of diabetes, smoking, education, stroke, coronary heart disease, BMI, SBP, TG, HDL-C, and LDL-C, multivariate logistic regression analysis found no association between $\mathrm{H}$. pylori infection and diabetes (OR 1.02, 95\% CI 0.881.18). Additionally, subgroup analysis indicated that $H$. pylori infection was significantly associated with increased risk of diabetes in the female group (OR 1.09, 95\% CI 1.08-1.09). Conclusions. No significant association was found between $H$. pylori infection and diabetes. However, the subgroup analysis suggested that $H$. pylori infection was possibly associated with increased risk of diabetes among females. Future cohort studies are needed to verify this association in females and to address possible implication in the prevention of diabetes.

\section{Introduction}

Diabetes has become a major public health problem with rapidly increasing prevalence worldwide. According to the International Diabetes Federation, 425 million people worldwide were estimated to have diabetes in 2017 [1]. A recent national survey revealed that the prevalence of diabetes in China was $10.9 \%$ in 2013 [2]. It is worthy of note that 114 million people in China were estimated to have diabetes in 2017, which means China has the world's largest diabetes epidemic [3]. Under this circumstance, identification of specific risk factors of diabetes in China will be of particular significance.

Helicobacter pylori (H. pylori) infection has been identified as a public health threat worldwide; approximately $50 \%$ of the world population is estimated to be infected with $H$. pylori [4]. Its prevalence has been demonstrated to range considerably according to socioeconomic status and tends to be more serious in developing countries $[4,5]$. In China, the prevalence of $H$. pylori infection ranges from $28.0 \%$ to 
$73.3 \%$ in different regions of the country $[6,7]$. H. pylori can commonly lead to gastrointestinal complications, including chronic gastritis, peptic ulcer, gastric adenocarcinoma, and mucosa-associated lymphoid tissue lymphoma [8, 9]. Notably, numerous studies have revealed significant association between $H$. pylori infection and extragastrointestinal conditions, such as cardiovascular disease, autoimmune disease, neurological disease, hyperemesis gravidarum during pregnancy, and metabolic disorders [10-14]. In particular, $H$. pylori infection is suspected to be associated with diabetes, which has become an intriguing field in recent years $[15,16]$.

However, existing studies addressing the association between $H$. pylori infection and diabetes provided conflicting results [17-21]. If $H$. pylori infection contributes to the incidence of diabetes, eradication of $H$. pylori infection may play an important role in diabetes prevention and control. Therefore, the objective of the current study was to examine the association between $H$. pylori infection and diabetes in a large medical checkup population in China.

\section{Methods}

2.1. Study Population. This was a cross-sectional study, using existing health checkup data from participants who attended routine annual health checkups at Beijing MJ Health Screening Center (Beijing, China) during 2017-2018. The health checkup data included anthropometric parameters, serum biochemical indices, and ${ }^{13} \mathrm{C}$-urea breath test. Meanwhile, demographic characteristics, self-reported lifestyle, and medical conditions were collected by a questionnaire during the health checkup. We excluded subjects aged $<18$ years and those with missing data on $H$. pylori infection, diabetes, family history of diabetes, hypertension, smoking, BMI, systolic blood pressure (SBP), diastolic blood pressure (DBP), total cholesterol (TC), triglyceride (TG), high-density lipoprotein cholesterol (HDL-C), low-density lipoprotein cholesterol (LDL-C), FPG, or HbA1c. This study has been approved by the Institutional Review Board of Peking University Health Science Center (ID of the approval: IRB00001052-19077). Individual informed consent was waived, as only anonymized data were used in this study.

2.2. Diagnosis of Diabetes. In the current study, diabetes was defined according to the American Diabetes Association criteria as meeting any of the following criteria [22]: (i) FPG $\geq 7.0 \mathrm{mmol} / \mathrm{L}$; (ii) $\mathrm{HbAlc} \geq 6.5 \%$; and (iii) self-reported physician diagnosis of diabetes or use of antidiabetic medication. Previous studies have confirmed that self-reported diabetes is a valid method to evaluate people's diabetes status in a Chinese and other population [23-25]. Fasting plasma glucose (FPG) was measured using the hexokinase method (Cobas 8000 modular analyzer, Roche Diagnostics). HbA1c was assayed using the ion-exchange high-performance liquid chromatography method (G8 HPLC Analyzer, Tosoh).

2.3. Establishment of $H$. pylori Infection. The ${ }^{13} \mathrm{C}$-urea breath test was applied to diagnose $H$. pylori infection. After overnight fasting for at least $8 \mathrm{~h}$, participants were required to take $75 \mathrm{mg}$ of ${ }^{13} \mathrm{C}$-urea (Urea- ${ }^{13} \mathrm{C}$ Capsule Breath Test Kit,
HEADWAY) after providing the initial baseline breath sample. We collected the second breath sample after half an hour. The samples were detected using a ${ }^{13} \mathrm{C}$-urea breath test analyzer (HCBT-01, HEADWAY). H. pylori infection was defined as positive if the difference between the two samples exceeded 4.0 parts per 1,000 of ${ }^{13} \mathrm{CO}_{2}$. Otherwise, it was considered negative.

2.4. Assessment of Covariates. Demographic characteristics (age, sex), lifestyle, and medical conditions were collected by a self-reported questionnaire. Level of education was divided into two categories according to whether individual participant received a college education (education time $\leq 12$ or $>12$ years). Smoking was classified as never smoker, former smoker, and current smoker. Family history of diabetes, stroke, and coronary heart disease were divided into two categories as yes or no. Standard laboratory methods were applied to obtain TC, TG, LDL-C, and HDL-C. Physical examinations, including height, weight, and blood pressure (BP), were performed by trained physicians. BMI was calculated as weight in kilograms divided by height squared in meters. BP was measured with a regular mercury sphygmomanometer on the right arm while seated after resting for at least $5 \mathrm{~min}$. Hypertension was defined as meeting any of the following criteria: (i) systolic blood pressure (SBP) $\geq 140 \mathrm{mmHg}$; (ii) diastolic blood pressure (DBP) $\geq 90 \mathrm{mmHg}$; and (iii) self-reported physician diagnosis of hypertension or use of antihypertensive medication.

2.5. Statistical Analysis. Characteristics of the participants are described as mean \pm SD for continuous variables with a normal distribution, median (interquartile range, IQR) for continuous variables with a skewed distribution, and percentages for categorical variables. Characteristics of participants across $H$. pylori status were compared using Student's $t$-test, Wilcoxon rank test, or $\chi^{2}$ test. To examine the association between $H$. pylori infection and diabetes, multivariate logistic regression analysis was performed to estimate adjusted odds ratios (ORs) and 95\% confidence intervals (CIs). Age- and sex- adjusted model (model 1) was performed first. Then, family history of diabetes (yes or no), smoking status (never smoker, former smoker, and current smoker), and level of education ( $\leq 12$ or $>12$ years) were further added into model 2 . Finally, we added potential risk factors of diabetes and confounders, including stroke (yes or no), coronary heart disease (yes or no), BMI (continuous variable), SBP (per $5 \mathrm{mmHg}$, continuous variable), TG (continuous variable), HDL-C (continuous variable), and LDL-C (continuous variable) into model 3 , to further evaluate the association between $H$. pylori infection and diabetes.

Subgroup analysis was performed using characteristics including age ( $<60$ or $\geq 60$ years old), sex (male or female), family history of diabetes (yes or no), hypertension (yes or no), smoking status (never smoker, former smoker, and current smoker), level of education ( $\leq 12$ or $>12$ years), BMI $\left(<24\right.$ or $\left.\geq 24 \mathrm{~kg} / \mathrm{m}^{2}\right)$, TG $(<1.7$ or $\geq 1.7 \mathrm{mmol} / \mathrm{L})$, HDL-C $(<1.0$ or $\geq 1.0 \mathrm{mmol} / \mathrm{L})$, and LDL-C $(\leq 4.1$ or $>4.1 \mathrm{mmol} / \mathrm{L})$ in the fully adjusted model (model 3 ). Alongside the grouping variable, other variables were used as adjustment 
TABLE 1: Characteristics of participants according to the status of $H$. pylori infection ${ }^{\dagger}$.

\begin{tabular}{|c|c|c|c|}
\hline Characteristics & H. pylori negative $(N=9,622)$ & H. pylori positive $(N=3,775)$ & $p$ value \\
\hline Age (years) & $43.8 \pm 12.2$ & $43.8 \pm 11.9$ & 0.96 \\
\hline Male, $n(\%)$ & $5,168(53.7)$ & $2,251(59.6)$ & $<0.001$ \\
\hline Diabetes, $n(\%)$ & $753(7.83)$ & $335(8.87)$ & 0.05 \\
\hline Family history of diabetes, $n(\%)$ & $1,787(18.6)$ & $641(17.0)$ & 0.03 \\
\hline Hypertension, $n(\%)$ & $1,571(16.3)$ & $720(19.1)$ & $<0.001$ \\
\hline Smoking status, $n(\%)$ & & & $<0.001$ \\
\hline Never smoker & $7,483(77.8)$ & $2,740(72.6)$ & \\
\hline Former smoker & $433(4.50)$ & $178(4.72)$ & \\
\hline Current smoker & $1,706(17.7)$ & $857(22.7)$ & \\
\hline Level of education, $n(\%)$ & & & $<0.001$ \\
\hline$\leq 12$ years & $459(4.77)$ & $279(7.39)$ & \\
\hline$>12$ years & $5,710(59.3)$ & $2,070(54.8)$ & \\
\hline Missing & $3,453(35.9)$ & $1,426(37.8)$ & \\
\hline BMI $\left(\mathrm{kg} / \mathrm{m}^{2}\right)$ & $23.9 \pm 3.44$ & $24.5 \pm 3.50$ & $<0.001$ \\
\hline $\mathrm{SBP}(\mathrm{mmHg})$ & $117 \pm 14.8$ & $118 \pm 15.3$ & $<0.001$ \\
\hline DBP (mmHg) & $72.1 \pm 10.8$ & $73.1 \pm 11.1$ & $<0.001$ \\
\hline $\mathrm{TC}(\mathrm{mmol} / \mathrm{L})$ & $4.63 \pm 0.89$ & $4.68 \pm 0.90$ & 0.004 \\
\hline TG (mmol/L) & $1.39 \pm 1.03$ & $1.48 \pm 1.12$ & $<0.001$ \\
\hline $\mathrm{HDL}-\mathrm{C}(\mathrm{mmol} / \mathrm{L})$ & $1.43 \pm 0.40$ & $1.38 \pm 0.38$ & $<0.001$ \\
\hline LDL-C (mmol/L) & $3.04 \pm 0.81$ & $3.10 \pm 0.82$ & $<0.001$ \\
\hline $\mathrm{FPG}(\mathrm{mmol} / \mathrm{L})$ & $5.62 \pm 1.00$ & $5.71 \pm 1.16$ & $<0.001$ \\
\hline HbAlc (\%) & $5.60 \pm 0.61$ & $5.64 \pm 0.71$ & 0.008 \\
\hline
\end{tabular}

${ }^{\dagger}$ Characteristics of participants across H. pylori status were compared using Student's $t$-test, Wilcoxon rank test, or $\chi^{2}$ test. BMI: body mass index; SBP: systolic blood pressure; DBP: diastolic blood pressure; TC: total cholesterol; TG: triglyceride; HDL-C: high-density lipoprotein cholesterol; LDL-C: low-density lipoprotein cholesterol; FPG: fasting plasma glucose. Data are mean \pm standard deviation or $n$ (\%).

variables. Statistical analysis was conducted by SAS 9.2 (SAS Institute, Cary, NC, USA). Two-tailed $p$ values $<0.05$ were considered statistically significant.

\section{Results}

A total of 13,397 participants (5,978 female and 7,419 male) were included in this study. The mean age of the participants was $43.8 \pm 12$ years. The prevalence of $H$. pylori infection and diabetes was $28.2 \%$ and $8.1 \%$, respectively. The prevalence of diabetes was higher among $H$. pylori-positive participants compared to their counterparts $(8.9 \%$ vs. $7.8 \%, p=0.05)$. Table 1 presented the characteristics of the participants across $H$. pylori status. Among all the participants, $18.1 \%$ have a family history of diabetes. Participants who had $H$. pylori infection were more likely to be males, smokers, and hypertensive with lower educational level and also had higher levels of BMI, SBP, DBP, TG, TC, LDL-C, FPG, and HbA1c, but lower level of HDL-C.

3.1. Associations between $H$. pylori Infection and Diabetes. Table 2 displayed the results of different models in the multivariate logistic regression analysis. The unadjusted OR for the association between $H$. pylori infection and diabetes was 1.15 (95\% CI 1.00-1.31; $p=0.05$ ). After adjustment for age
TABle 2: Association between $H$. pylori infection and diabetes.

\begin{tabular}{lcccc}
\hline Model $^{\dagger}$ & $\begin{array}{c}\text { H. pylori } \\
\text { negative }\end{array}$ & $\begin{array}{c}\text { H. pylori } \\
\text { positive }\end{array}$ & OR (95\% CI) & p value \\
\hline $\begin{array}{l}\text { Unadjusted } \\
\text { model }\end{array}$ & $753(7.83)$ & $335(8.87)$ & $1.15(1.00-1.31)$ & 0.05 \\
Model 1 & $753(7.83)$ & $335(8.87)$ & $1.14(0.99-1.32)$ & 0.07 \\
Model 2 & $753(7.83)$ & $335(8.87)$ & $1.10(0.95-1.28)$ & 0.19 \\
Model 3 & $753(7.83)$ & $335(8.87)$ & $1.02(0.88-1.18)$ & 0.81 \\
\hline
\end{tabular}

${ }^{\dagger}$ Model 1: adjusted for age and sex. Model 2: model $1+$ family history of diabetes, smoking status, and level of education. Model 3: model $2+$ stroke, coronary heart disease, BMI, SBP, TG, HDL-C, and LDL-C. Data are $n(\%)$.

and sex, $H$. pylori infection was associated with an increased risk of diabetes (OR 1.14, 95\% CI 0.99-1.32) but without statistical significance $(p=0.07)$. Additional adjustment for family history of diabetes, smoking, and education obtained attenuated the result (OR 1.10, 95\% CI 0.95-1.28; $p=0.19$ ). In the fully adjusted analysis, $H$. pylori infection was not significantly associated with diabetes (OR $1.02,95 \%$ CI 0.88 $1.18 ; p=0.81$ ), after further adjustment for stroke, coronary heart disease, BMI, SBP, TG, HDL-C, and LDL-C.

3.2. Subgroup Analysis for the Association between H. pylori Infection and Diabetes. The association of $H$. pylori infection 
TABLE 3: Subgroup analysis of the association between $H$. pylori infection and diabetes ${ }^{\dagger}$.

\begin{tabular}{|c|c|c|c|c|c|}
\hline Subgroup & $\mathrm{n}$ & H. pylori negative & H. pylori positive & OR $(95 \% \mathrm{CI})$ & $p$ value \\
\hline All & 13,397 & $9,622(71.8)$ & $3,775(28.2)$ & $1.02(0.88-1.18)$ & 0.81 \\
\hline \multicolumn{6}{|l|}{ Age (years) } \\
\hline$<60$ & 11,941 & $482(5.63)$ & $226(6.70)$ & $1.00(0.84-1.20)$ & 0.97 \\
\hline$\geq 60$ & 1,456 & $271(25.7)$ & $109(27.1)$ & $1.01(0.76-1.34)$ & 0.93 \\
\hline \multicolumn{6}{|l|}{ Sex } \\
\hline Male & 7,419 & $538(10.4)$ & $245(10.9)$ & $1.00(0.84-1.20)$ & 0.97 \\
\hline Female & 5,978 & $215(4.83)$ & $90(5.91)$ & $1.09(1.08-1.09)$ & $<0.001$ \\
\hline \multicolumn{6}{|c|}{ Family history of diabetes } \\
\hline Yes & 2,428 & $224(12.5)$ & $94(14.7)$ & $1.03(0.76-1.39)$ & 0.80 \\
\hline No & 10,969 & $529(6.75)$ & $241(7.69)$ & $1.02(0.86-1.22)$ & 0.87 \\
\hline \multicolumn{6}{|l|}{ Hypertension } \\
\hline Yes & 2291 & $339(21.6)$ & $166(23.1)$ & $1.16(0.92-1.46)$ & 0.52 \\
\hline No & 11,106 & $414(5.14)$ & $169(5.53)$ & $0.94(0.77-1.14)$ & 0.21 \\
\hline \multicolumn{6}{|l|}{ Smoking status } \\
\hline Never smoker & 10,223 & $457(6.11)$ & $189(6.90)$ & $1.03(0.84-1.25)$ & 0.80 \\
\hline Former smoker & 661 & $68(15.7)$ & $27(15.2)$ & $1.02(0.59-1.74)$ & 0.96 \\
\hline Current smoker & 2,563 & $228(13.4)$ & $119(13.9)$ & $1.03(0.79-1.35)$ & 0.81 \\
\hline \multicolumn{6}{|l|}{ Level of education } \\
\hline$\leq 12$ years & 738 & 99 (21.6) & $57(20.4)$ & $0.88(0.58-1.33)$ & 0.54 \\
\hline$>12$ years & 7,780 & $374(6.55)$ & $149(7.20)$ & $1.05(0.85-1.31)$ & 0.64 \\
\hline Missing & 4,879 & $280(8.11)$ & $129(9.05)$ & $1.00(0.78-1.27)$ & 0.97 \\
\hline \multicolumn{6}{|l|}{ BMI $\left(\mathrm{kg} / \mathrm{m}^{2}\right)$} \\
\hline$<24$ & 6,926 & $215(4.14)$ & $75(4.34)$ & $1.10(0.82-1.47)$ & 0.54 \\
\hline$\geq 24$ & 6,471 & $538(12.2)$ & $260(12.7)$ & $1.00(0.84-1.18)$ & 0.96 \\
\hline \multicolumn{6}{|l|}{$\mathrm{TG}(\mathrm{mmol} / \mathrm{L})$} \\
\hline$<1.7$ & 10,040 & $418(5.70)$ & $156(5.75)$ & $0.91(0.74-1.12)$ & 0.40 \\
\hline$\geq 1.7$ & 3,357 & $335(14.6)$ & $179(16.9)$ & $1.16(0.93-1.44)$ & 0.18 \\
\hline \multicolumn{6}{|l|}{ HDL-C (mmol/L) } \\
\hline$<1.0$ & 1,822 & $182(14.8)$ & $80(13.6)$ & $0.86(0.63-1.18)$ & 0.35 \\
\hline$\geq 1.0$ & 11,575 & $571(6.81)$ & $255(8.00)$ & $1.08(0.91-1.28)$ & 0.38 \\
\hline \multicolumn{6}{|l|}{ LDL-C (mmol/L) } \\
\hline$\leq 4.1$ & 12,115 & $659(7.55)$ & $283(8.36)$ & $0.99(0.84-1.16)$ & 0.87 \\
\hline$>4.1$ & 1,282 & $94(10.5)$ & $52(13.3)$ & $1.16(0.78-1.71)$ & 0.47 \\
\hline
\end{tabular}

${ }^{\dagger}$ All the subgroup analyses were adjusted for age, sex, family history of diabetes, hypertension, smoking status, level of education, BMI, TG, HDL-C, and LDL-C. BMI: body mass index; TG: triglyceride; HDL-C: high-density lipoprotein cholesterol; LDL-C: low-density lipoprotein cholesterol. Data are $n$ (\%).

with diabetes was investigated according to different subgroups, including age, sex, family history of diabetes, hypertension, smoking, education, BMI, TG, HDL-C, and LDL-C (Table 3). H. pylori infection was significantly associated with diabetes among females (OR 1.09, 95\% CI 1.08-1.09; $p<$ $0.001)$. For all the other subgroups, no significant associations were found between $H$. pylori infection and diabetes (all $p>0.05$ ).

\section{Discussion}

In the present study, we found no significant association between $H$. pylori infection and prevalent diabetes after adjustment for risk factors of diabetes and potential confounders. However, subgroup analysis did find an association between $H$. pylori infection and increased risk of prevalent diabetes among females.

The relationship between $H$. pylori infection and diabetes remained controversial for the last years. Several studies supported our results on the lack of association between $H$. pylori infection and diabetes $[17,20,26]$. In contrast, others found $H$. pylori infection to be positively associated with diabetes $[19,21,27,28]$. A meta-analysis that included 41 casecontrol studies concluded that $H$. pylori infection could be a potential risk factor for type 2 diabetes, while among them, two studies conducted in China both showed a negative result [29]. Nevertheless, few of these previous studies had adjusted family history of diabetes in their analysis. Since there has been compelling evidence supporting the strong role of genetics in the development of diabetes [30, 31], the 
relationship between $H$. pylori infection and diabetes cannot be truly clarified without adjusting family history of diabetes.

Although there is no strong evidence about $H$. pylori infection resulting in diabetes and the underlying mechanism is not clear, it is biologically plausible. Some researchers believed that chronic inflammation and insulin resistance induced by $H$. pylori may increase the risk for diabetes [32, 33]. H. pylori colonization can trigger inflammation responses. Neutrophil cells penetrate the gastrointestinal mucus at the initial phases of infection. Then, at the chronic phase of infection, those cells are replaced by monocyte instead. Monocyte is capable of producing a variety of inflammatory cytokines (mainly including tumor necrosis factor- $\alpha$, interleukin, and C-reactive protein) that, in addition to exerting local effects, can also be released on other tissues and organs and ultimately cause an increase in extragastrointestinal conditions $[34,35]$. Those inflammatory cytokines probably contribute to diabetes by causing insulin resistance and are in turn intensified in the presence of hyperglycemia to promote long-term complications of diabetes [36]. Several studies considered these inflammatory cytokines as the connection between $\mathrm{H}$. pylori and diabetes $[37,38]$, and some found the connection with sex difference $[39,40]$, while others found that the connection adds little to the association between $H$. pylori and diabetes once classical risk factors for diabetes have been accounted for [41, 42].

More studies are necessary to elucidate the pathogenesis of $H$. pylori resulting in diabetes. It is worth to mention that results of some studies have indicated that $H$. pylori cytotoxin-associated gene A (CagA), which has been identified as a possible virulence marker of $H$. pylori [43], induces more severe inflammatory responses [44]. Future studies should examine the effect of CagA positivity in the process of $H$. pylori-induced diabetes. Besides, the duration of $H$. pylori infection is also worth of concern. Compared to subjects with short-term infection, those with more longstanding infection may have more severe mucosal damage and inflammatory responses and thereby develop insulin resistance.

Notably, growing evidence showed that inflammation process and insulin resistance were highly depending on sex and sex hormone status throughout the lifetime [45-47]. A study had identified a direct interaction between sex hormones and microbial exposures and showed that microbiome manipulations can provoke a testosterone-dependent effect on inflammation and autoimmunity in a genetically high-risk rodent model. The transfer of gut microbiota from adult males to immature females altered the recipient's microbiota and testosterone level, resulting in metabolomic changes, reduced islet inflammation and autoantibody production, and ultimately robust type 1 diabetes protection $[48,49]$. In the present study, we found that $H$. pylori infection was associated with increased risk of diabetes only among females. Based on the evidence provided by previous studies, we speculated that the sex discrepancy of diabetes susceptibility found in our study was likely due to the interaction between sex hormones and $H$. pylori-induced inflammation.

Increased inflammation induced by $H$. pylori infection could lead to insulin resistance and diabetes through a sexdimorphic hypothalamic insulin action. Studies using a rat model have proved that inflammation was a potential cause for impaired insulin action within the hypothalamus [50]. Specifically, systemic inflammation induced by $H$. pylori infection could increase the expression of proinflammatory cytokines such as interleukin- 6 and tumor necrosis factor- $\alpha$. Those cytokines were able to act on the hypothalamus and result in hypothalamic inflammation [51]. Hypothalamic inflammation could reduce insulin signaling and was an important cause of the disrupted neuroendocrine control of metabolism that resulted in systemic insulin resistance [52, 53], whereas insulin signaling and action were found to be more disrupted in female rats than in male rats [50]. The underlying mechanism was the modulation caused by brain estrogen signaling, potentially mediated by estrogen effects on ER $\alpha$ in various regions of the hypothalamus $[54,55]$. The fundamental sex difference in central insulin signaling was not only found in animals but also corroborated in humans $[54,56]$. The joint effect of $H$. pylori-induced inflammation and sex hormones mentioned above might explain for the more pronounced insulin resistance among females and finally made them more predisposed to diabetes compared with males.

The present study had several strengths. First, we fully adjusted potential confounders and risk factors of diabetes in the multivariate analysis, which made the results more reliable and convincing. Few of the previous studies, especially those that found a significant association between $H$. pylori and diabetes, included family history of diabetes into their multivariate regression models. This probably biased the results. Second, the diagnosis of $H$. pylori infection in our study was established by the ${ }^{13} \mathrm{C}$-urea breath test rather than serologic testing. Previous studies have found that there is a need for an optimization of ${ }^{13} \mathrm{C}$-urea breath test threshold levels, while the ${ }^{13} \mathrm{C}$-urea breath test is still believed to be the most widely available and accurate noninvasive test for $H$. pylori diagnosis compared with serologic testing, which was commonly used in previous studies [57, 58]. Despite the strengths, there were still some limitations in our study. First, due to the cross-sectional design, causal relationship between $H$. pylori infection and diabetes could not be determined. Larger-scale prospective cohort studies are required to investigate the association between $H$. pylori infection and risk of incident diabetes. Second, possible confounding factors, i.e., polycystic ovary syndrome, total energy intake, and physical activity, were not adjusted in the regression analysis, as they were not precisely measured in the health checkup population. However, we adjusted BMI in our analysis, which to some extent could represent the effect of total energy intake and physical activity. Granted the possible confounding factors and the small effect sizes we calculated, the observed association between $H$. pylori infection and diabetes among females in the present study may be statistically but not clinically significant [59]. Only after critically examining the clinical significance and relevance of this association should it be applied to clinical situations.

\section{Conclusions}

In summary, we found no significant association between $H$. pylori infection and diabetes. However, an increased risk of 
prevalent diabetes was found among females with $H$. pylori infection. Large-scale, well-designed prospective studies are needed to investigate if $H$. pylori plays an etiological role in the development of diabetes.

\section{Data Availability}

All data used to support the findings of this study may be released upon application to the Meinian Institute of Health (Beijing, China), which can be contacted through Prof. Yi Ning (email: yi.ning@meinianresearch.com).

\section{Conflicts of Interest}

The authors declare that there was no potential competing interest.

\section{Authors' Contributions}

SM and BW wrote the manuscript. BW, CJ, and YN designed the study. YM, MT, and CJ researched the data. SM, BW, JV, LL, and YN contributed to the discussion and reviewed the manuscript. Sailimai Man and Yuan Ma should be considered joint first authors. Sailimai Man and Yuan Ma contributed equally to this work.

\section{Acknowledgments}

The present study was funded by National Natural Science Foundation of China (No. 91846303) and Ministry of Science and Technology of China (No. 2020YFC2003400).

\section{References}

[1] International Diabetes Federation, IDF Diabetes Atlas, International Diabetes Federation, Brussels, Belgium, 8th edition, 2017.

[2] L. Wang, P. Gao, M. Zhang et al., "Prevalence and ethnic pattern of diabetes and prediabetes in China in 2013," Journal of the American Medical Association, vol. 317, no. 24, pp. 25152523, 2017.

[3] Y. Xu, L. Wang, J. He et al., "Prevalence and control of diabetes in Chinese adults," Journal of the American Medical Association, vol. 310, no. 9, pp. 948-959, 2013.

[4] M. Zamani, F. Ebrahimtabar, V. Zamani et al., "Systematic review with meta-analysis: the worldwide prevalence of Helicobacter pylori infection," Alimentary Pharmacology \& Therapeutics, vol. 47, no. 7, pp. 868-876, 2018.

[5] I. Thung, H. Aramin, V. Vavinskaya et al., "Review article: the global emergence of Helicobacter pylori antibiotic resistance," Alimentary Pharmacology \& Therapeutics, vol. 43, no. 4, pp. 514-533, 2016.

[6] S. Peng, Y. Cui, Y. L. Xiao et al., "Prevalence of erosive esophagitis and Barrett's esophagus in the adult Chinese population," Endoscopy, vol. 41, no. 12, pp. 1011-1017, 2009.

[7] Z. Li, D. Zou, X. Ma et al., "Epidemiology of peptic ulcer disease: endoscopic results of the systematic investigation of gastrointestinal disease in China," The American Journal of Gastroenterology, vol. 105, no. 12, pp. 2570-2577, 2010.

[8] H. Shmuely, M. Wattad, A. Solodky, J. Yahav, Z. Samra, and N. Zafrir, "Association of Helicobacter pylori with coronary artery disease and myocardial infarction assessed by myocardial perfusion imaging," The Israel Medical Association Journal, vol. 16, no. 6, pp. 341-346, 2014.

[9] J. Kountouras, S. A. Polyzos, M. Doulberis et al., "Potential impact of Helicobacter pylori-related metabolic syndrome on upper and lower gastrointestinal tract oncogenesis," Metabolism, vol. 87, pp. 18-24, 2018.

[10] M. Waluga, M. Kukla, M. Zorniak, A. Bacik, and R. Kotulski, "From the stomach to other organs: Helicobacter pylori and the liver," World Journal of Hepatology, vol. 7, no. 18, pp. 2136-2146, 2015.

[11] F. Franceschi, A. Gasbarrini, S. A. Polyzos, and J. Kountouras, "Extragastric diseases and Helicobacter pylori," Helicobacter, vol. 20, Suppl 1, pp. 40-46, 2015.

[12] S. A. Polyzos and J. Kountouras, "Novel advances in the association between Helicobacter pylori infection, metabolic syndrome, and related morbidity," Helicobacter, vol. 20, no. 6, pp. 405-409, 2015.

[13] A. S. Bakka and B. A. Salih, "Prevalence of Helicobacter pylori infection in asymptomatic subjects in Libya," Diagnostic Microbiology and Infectious Disease, vol. 43, no. 4, pp. 265268, 2002.

[14] Q. X. Ng, N. Venkatanarayanan, M. L. Z. Q. De Deyn, C. Y. X. Ho, Y. Mo, and W.-S. Yeo, "A meta-analysis of the association betweenHelicobacter pylori(H. pylori) infection and hyperemesis gravidarum," Helicobacter, vol. 23, no. 1, 2018.

[15] E. Goni and F. Franceschi, "Helicobacter pylori and extragastric diseases," Helicobacter, vol. 21, Suppl 1, pp. 45-48, 2016.

[16] Y. Chen and M. J. Blaser, "Association between gastric Helicobacter pylori colonization and glycated hemoglobin levels," The Journal of Infectious Diseases, vol. 205, no. 8, pp. 1195$1202,2012$.

[17] N. Wawro, U. Amann, J. Butt et al., "Helicobacter pylori seropositivity: prevalence, associations, and the impact on incident metabolic diseases/risk factors in the population-based KORA study," Frontiers in Public Health, vol. 7, p. 96, 2019.

[18] M. Zhou, J. Liu, Y. Qi et al., "The association between Helicobacter pylori seropositivity and risk of new-onset diabetes: a prospective cohort study," Diabetologia, vol. 61, no. 2, pp. 300-307, 2018.

[19] Z. Wan, L. Song, L. Hu et al., "Helicobacter pylori infection is associated with diabetes among Chinese adults," Journal of Diabetes Investigation, vol. 11, no. 1, pp. 199-205, 2020.

[20] H. H.-X. Xia, N. J. Talley, E. P. Y. Kam, L. J. Young, J. Hammer, and M. Horowitz, "Helicobacter pylori infection is not associated with diabetes mellitus, nor with upper gastrointestinal symptoms in diabetes mellitus," The American Journal of Gastroenterology, vol. 96, no. 4, pp. 1039-1046, 2001.

[21] C. Y. Jeon, M. N. Haan, C. Cheng et al., "Helicobacter pylori infection is associated with an increased rate of diabetes," Diabetes Care, vol. 35, no. 3, pp. 520-525, 2012.

[22] American Diabetes Association, "2. Classification and diagnosis of Diabetes:Standards of medical care in diabetes-2018," Diabetes Care, vol. 41, Supplement 1, pp. S13-S27, 2017.

[23] X. Yuan, T. Liu, L. Wu, Z. Y. Zou, and C. Li, "Validity of selfreported diabetes among middle-aged and older Chinese adults: the China Health and Retirement Longitudinal Study," BMJ Open, vol. 5, no. 4, article e006633, 2015.

[24] J. M. Jackson, T. A. DeFor, A. L. Crain et al., "Validity of diabetes self-reports in the Women's Health Initiative," Menopause, vol. 21, no. 8, pp. 861-868, 2014. 
[25] S. Pastorino, M. Richards, R. Hardy et al., "Validation of selfreported diagnosis of diabetes in the 1946 British birth cohort," Primary Care Diabetes, vol. 9, no. 5, pp. 397-400, 2015.

[26] T. Y. Yu, J. N. Wei, C. H. Kuo et al., "The impact of gastric atrophy on the incidence of diabetes," Scientific Reports, vol. 7, no. 1, article 39777, 2017.

[27] Y. Y. Chen, W. H. Fang, C. C. Wang et al., "Helicobacter pylori infection increases risk of incident metabolic syndrome and diabetes: a cohort study," PLoS One, vol. 14, no. 2, article e0208913, 2019.

[28] X. Han, Y. Li, J. Wang et al., "Helicobacter pylori infection is associated with type 2 diabetes among a middle- and old-age Chinese population," Diabetes/Metabolism Research and Reviews, vol. 32, no. 1, pp. 95-101, 2016.

[29] K. Mansori, Y. Moradi, S. Naderpour et al., "Helicobacter pylori infection as a risk factor for diabetes: a meta-analysis of case-control studies," BMC Gastroenterology, vol. 20, no. 1, p. 77, 2020.

[30] P. Poulsen, K. O. Kyvik, A. Vaag, and H. Beck-Nielsen, "Heritability of type II (non-insulin-dependent) diabetes mellitus and abnormal glucose tolerance-a population-based twin study," Diabetologia, vol. 42, no. 2, pp. 139-145, 1999.

[31] P. Poulsen, L. G. Grunnet, K. Pilgaard et al., "Increased risk of type 2 diabetes in elderly twins," Diabetes, vol. 58, no. 6, pp. 1350-1355, 2009.

[32] S. A. Hosseininasab Nodoushan and A. Nabavi, "The interaction of Helicobacter pylori infection and type 2 diabetes mellitus," Advanced Biomedical Research, vol. 8, no. 1, p. 15, 2019.

[33] C. He, Z. Yang, and N. H. Lu, "Helicobacter pylori infection and diabetes: is it a myth or fact?," World Journal of Gastroenterology, vol. 20, no. 16, pp. 4607-4617, 2014.

[34] L. Jackson, J. Britton, S. A. Lewis et al., “A population-based epidemiologic study of Helicobacter pylori infection and its association with systemic inflammation," Helicobacter, vol. 14, no. 5, pp. 108-113, 2009.

[35] S. A. Hamed, N. F. Amine, G. M. Galal et al., "Vascular risks and complications in diabetes mellitus: the role of helicobacter pylori infection," Journal of Stroke and Cerebrovascular Diseases, vol. 17, no. 2, pp. 86-94, 2008.

[36] G. L. King, "The role of inflammatory cytokines in diabetes and its complications," Journal of Periodontology, vol. 79, no. 8s, pp. 1527-1534, 2008.

[37] B. Thorand, H. Löwel, A. Schneider et al., "C-reactive protein as a predictor for incident diabetes mellitus among middleaged men," Archives of Internal Medicine, vol. 163, no. 1, pp. 93-99, 2003.

[38] D. J. Freeman, J. Norrie, M. J. Caslake et al., "C-reactive protein is an independent predictor of risk for the development of diabetes in the West of Scotland Coronary Prevention Study," Diabetes, vol. 51, no. 5, pp. 1596-1600, 2002.

[39] T. S. Han, N. Sattar, K. Williams, C. Gonzalez-Villalpando, M. E. Lean, and S. M. Haffner, "Prospective study of Creactive protein in relation to the development of diabetes and metabolic syndrome in the Mexico City Diabetes Study," Diabetes Care, vol. 25, no. 11, pp. 2016-2021, 2002.

[40] S. McDonald, G. Maguire, N. Duarte, X. L. Wang, and W. Hoy, "C-reactive protein, cardiovascular risk, and renal disease in a remote Australian Aboriginal community," Clinical Science, vol. 106, no. 2, pp. 121-128, 2004.
[41] J. Krakoff, T. Funahashi, C. D. A. Stehouwer et al., "Inflammatory markers, adiponectin, and risk of type 2 diabetes in the Pima Indian," Diabetes Care, vol. 26, no. 6, pp. 1745-1751, 2003.

[42] E. Lontchi-Yimagou, E. Sobngwi, T. E. Matsha, and A. P. Kengne, "Diabetes mellitus and inflammation," Current Diabetes Reports, vol. 13, no. 3, pp. 435-444, 2013.

[43] H. Lu, Y. Yamaoka, and D. Y. Graham, "Helicobacter pylori virulence factors: facts and fantasies," Current Opinion in Gastroenterology, vol. 21, no. 6, pp. 653-659, 2005.

[44] N. Kaklikkaya, K. Cubukcu, F. Aydin et al., "Significance of cagA status and vacA subtypes of Helicobacter pylori in determining gastric histopathology: virulence markers of $\mathrm{H}$. pylori and histopathology," Journal of Gastroenterology and Hepatology, vol. 21, no. 6, pp. 1042-1047, 2006.

[45] J. L. Faulkner and E. J. Belin de Chantemele, "Sex hormones, aging and cardiometabolic syndrome," Biol Sex Differ, vol. 10, no. 1, p. 30, 2019

[46] M. Varghese, C. Griffin, and K. Singer, "The role of sex and sex hormones in regulating obesity-induced inflammation," Advances in Experimental Medicine and Biology, vol. 1043, pp. 65-86, 2017.

[47] D. C. Henstridge, J. Abildgaard, B. Lindegaard, and M. A. Febbraio, "Metabolic control and sex: a focus on inflammatorylinked mediators," British Journal of Pharmacology, 2018.

[48] J. G. M. Markle, D. N. Frank, S. Mortin-Toth et al., "Sex differences in the gut microbiome drive hormone-dependent regulation of autoimmunity," Science, vol. 339, no. 6123, pp. 1084-1088, 2013.

[49] E. A. Ivakine, S. M. Mortin-Toth, O. M. Gulban et al., "The idd4 locus displays sex-specific epistatic effects on type 1 diabetes susceptibility in nonobese diabetic mice," Diabetes, vol. 55, no. 12, pp. 3611-3619, 2006.

[50] C. Lindtner, T. Scherer, E. Zielinski et al., "Binge drinking induces whole-body insulin resistance by impairing hypothalamic insulin action," Science Translational Medicine, vol. 5, no. 170, article 170ra14, 2013.

[51] S. W. Coppack, "Pro-inflammatory cytokines and adipose tissue," The Proceedings of the Nutrition Society, vol. 60, no. 3, pp. 349-356, 2001.

[52] P. J. Klover, T. A. Zimmers, L. G. Koniaris, and R. A. Mooney, "Chronic exposure to interleukin-6 causes hepatic insulin resistance in mice," Diabetes, vol. 52, no. 11, pp. 2784-2789, 2003.

[53] J. J. Senn, P. J. Klover, I. A. Nowak et al., "Suppressor of cytokine signaling-3 (SOCS-3), a potential mediator of interleukin6-dependent insulin resistance in hepatocytes," The Journal of Biological Chemistry, vol. 278, no. 16, pp. 13740-13746, 2003.

[54] D. J. Clegg, L. M. Brown, S. C. Woods, and S. C. Benoit, "Gonadal hormones determine sensitivity to central leptin and insulin," Diabetes, vol. 55, no. 4, pp. 978-987, 2006.

[55] A. Kautzky-Willer, J. Harreiter, and G. Pacini, "Sex and gender differences in risk, pathophysiology and complications of type 2 diabetes mellitus," Endocrine Reviews, vol. 37, no. 3, pp. 278316, 2016.

[56] L. K. Burke, B. Doslikova, G. D'Agostino et al., "Sex difference in physical activity, energy expenditure and obesity driven by a subpopulation of hypothalamic POMC neurons," Molecular Metabolism, vol. 5, no. 3, pp. 245-252, 2016.

[57] T. Mock, R. Yatscoff, R. Foster et al., "Clinical validation of the Helikit: a 13C urea breath test used for the diagnosis of 
Helicobacter pylori infection," Clinical Biochemistry, vol. 32, no. 1, pp. 59-63, 1999.

[58] T. T. Perets, R. Gingold-Belfer, H. Leibovitzh et al., "Optimization of 13C-urea breath test threshold levels for the detection of Helicobacter pylori infection in a national referral laboratory," Journal of Clinical Laboratory Analysis, vol. 33, no. 2, article e22674, 2019.

[59] Q. X. Ng, A. Y.S. Soh, and W. S. Yeo, "Statistically but not clinically significant? Biomarkers in gastric cancer," Clinical Nutrition, vol. 37, no. 6, pp. 2292-2293, 2018. 